\title{
Observation de la végétation depuis l'espace
}

\author{
Jean-Christophe Calvet ${ }^{1}$, Éric Ceschia ${ }^{2}$, Dominique Courault ${ }^{3}$, \\ Hélène Dewaele', Yves Goulas ${ }^{4}$, Jordi Inglada ${ }^{2}$, Thuy Le Toan ${ }^{2}$, \\ Fabienne Maignan ${ }^{5}$ \\ 1 Centre national de recherches météorologiques, Météo-France, Toulouse \\ 2 Centre d'études spatiales de la biosphère, Cnes / Université Paul-Sabatier / \\ CNRS, Toulouse \\ 3 Environnement méditerranéen et modélisation des agro-hydro-systèmes, \\ Inra, Avignon \\ 4 Laboratoire de météorologie dynamique, École polytechnique / CNRS, \\ Palaiseau \\ 5 Laboratoire des sciences du climat et de l'environnement, \\ Institut Pierre-Simon Laplace, CNRS / CEA / UVSQ, Gif-sur-Yvette
}

L'arrivée de nouvelles données satellitaires en accès libre (notamment du programme européen Sentinel de Copernicus) fait avancer la caractérisation de l'occupation des sols et des cycles de l'eau et du carbone. La résolution spatiale décamétrique de ces données, disponibles à une fréquence élevée, permet de produire des variables biogéophysiques à l'échelle des parcelles agricoles. Des applications en agrométéorologie sont possibles, mais également pour la validation et l'amélioration des modèles des surfaces terrestres utilisés en météorologie et en climat. Deux nouvelles missions de l'Agence spatiale européenne, Biomass et Flex, dont les lancements sont programmés respectivement pour 2021 et 2022 , vont apporter des connaissances nouvelles sur la photosynthèse et le stockage de carbone par les forêts.

\section{Abstract}

\section{Observing vegetation from space}

New freely available satellite products (in particular from the European Copernicus Sentinel programme) are fostering progress in land cover mapping and in the monitoring of water and carbon cycles. These observations are available with a high sampling time and at a spatial resolution compatible with the size of most agricultural parcels. Agrometeorological applications are emerging and these data will also contribute to the validation and evolution of the land surface models used in weather forecast and climate models. Two new research missions from the European Space Agency, Biomass and Flex to be launched in 2021 et 2022, respectively, will tackle key knowledge gaps in photosynthesis and forest carbon storage.

jean-christophe.calvet@meteo.fr es terres agricoles et les forêts sont au cœur des problématiques de développement durable, parce qu'elles contribuent aux changements globaux via l'évolution de leurs états de surfaces (albédo, flux de chaleur...), en consommant des ressources en eau et en émettant (ou en absorbant) des gaz à effet de serre dont le $\mathrm{CO}_{2}$. Ces surfaces subissent aussi ces changements globaux, ce qui affecte notamment la production alimentaire. Pour maintenir des niveaux suffisants de production agricole et réduire les impacts environnementaux de ces agro-écosystèmes, il faut pouvoir quantifier et analyser spatialement l'évolution des rendements et des indicateurs environnementaux comme les bilans de carbone, mais aussi comprendre l'influence des conditions environnementales et de la gestion des cultures sur ces variables. Le système mondial d'observation du climat (Global Climate Observing System, GCOS) a proposé une liste de variables climatiques essentielles terrestres observables par télédétection. Parmi elles, six sont directement influencées par la végétation : l'albédo de surface, l'occupation des sols, la fraction de rayonnement actif pour la photosynthèse absorbée, l'indice de surface foliaire (LAI, pour Leaf Area Index), la biomasse aérienne (en particulier des forêts) et la température de surface. Les flux d'eau (évapotranspiration) ou de $\mathrm{CO}_{2}$ ne figurent pas aujourd'hui dans cette liste, car ils ne sont pas observables directement. Ils peuvent être obtenus en exploitant des complémentarités entre diverses observations satellitaires et en intégrant ces observations dans des modèles plus ou moins complexes, en particulier en utilisant des techniques d'assimilation de données.

\section{Utilisation des terres et gestion de l'eau}

En France, l'agriculture consomme aujourd'hui plus de $75 \%$ des ressources totales en eau. En région Provence-Alpes-Côte-d'Azur, les cultures irriguées représentent $70 \%$ des zones cultivées. Les besoins en eau varient selon les cultures. Par exemple, pour un cycle annuel complet de végétation, le besoin est d'environ $500 \mathrm{~mm}$ d'eau pour la production de légumes et de plus de $650 \mathrm{~mm}$ pour les vergers. Les épisodes de sécheresse récurrents des quinze dernières années ont entraîné dans certains secteurs des restrictions sur la quantité d'eau pouvant être utilisée pour l'irrigation, conduisant à des pertes de rendement significatives. Dans ce contexte, il est important de développer des méthodes qui permettent de mettre en œuvre une gestion spatiale et intégrée de l'eau afin de faire face à l'augmentation de la demande et à la diminution des ressources en eau. Autrement dit de gérer les ressources en eau afin de répondre aux objectifs d'un développement durable. 
La télédétection apporte des informations essentielles sur:

- les variations spatio-temporelles de nombreux paramètres hydrologiques clés pour les outils d'aide à la décision (volumes d'eau de surface, humidité des sols, stock d'eau total d'un bassinversant, évapotranspiration, détection des stress hydriques, etc.) ;

- les modifications de l'environnement dues aux activités humaines (par exemple l'occupation des sols, les types de culture, les interventions agricoles, etc.), à des résolutions spatiale et temporelle compatibles avec la gestion de la ressource en eau (voir encadré 1).

Ces observations sont utilisées dans des modèles de fonctionnement des cultures et des modèles hydrologiques selon deux méthodes : forçage et inversion du modèle. Le forçage du modèle consiste à remplacer directement certaines variables d'entrée du modèle par le produit issu de la télédétection. L'inversion consiste à caler au mieux la valeur des paramètres du modèle de façon à reproduire les observations satellitaires. L'estimation par les modèles de la production agricole et des ressources en eau est améliorée. De nombreuses observations spatiales sont d'ores et déjà disponibles sur un large domaine spatial et temporel. Ces données fournissent des informations souvent complémentaires selon le domaine de longueur d'onde utilisé.

\section{L'occupation des sols}

L'objectif principal de la cartographie de l'occupation des sols est de comprendre les évolutions et les tendances des typologies de zones au cours du temps (par exemple, le remplacement d'une zone de forêt en zone artificialisée) afin d'en expliquer les causes et d'en prévoir les conséquences. Les cartes d'occupation des sols constituent un outil essentiel pour l'aménagement du territoire ou l'analyse des impacts du changement climatique. Elles sont aussi utilisées en entrée des modèles des surfaces terrestres utilisés en hydrologie, dans l'étude du climat, et pour la description des cycles de l'eau et du carbone. L'occupation des sols a été identifiée comme l'une des variables climatiques essentielles (Bojinski et al., 2014) par le GCOS.

Le terme "occupation des sols" englobe souvent deux aspects différents : l'occupation des sols à proprement parler et l'utilisation des terres. Dans certaines applications, i est intéressant d'obtenir une vue physionomique du terrain. On parle alors d'occupation des sols (land cover) : on cherche à distinguer les éléments qui forment la structure du paysage sans essayer d'en identifier la fonction. D'un autre côté, il est souvent nécessaire $d^{\prime}$ adopter un point de vue anthropique sur le paysage, afin de prendre en compte la fonction ou le type d'usage qui est fait de l'espace. On parle alors d'utilisation des terres (land use). Cette distinction, qui est très importante du point de vue de l'utilisateur des cartes, n'a pas beaucoup d'incidence sur les méthodologies employées pour leur production.

Pour une prise en compte précise des évolutions, notamment de la végétation, il est nécessaire de mettre à jour les cartes d'occupation des sols de façon fréquente (tous les ans ou même tous les trois mois). La télédétection spatiale constitue un outil incontournable pour ce faire et tout particulièrement les données à hautes résolutions spatiale et temporelle comme celles qui commencent à être fournies par les satellites du programme européen Copernicus. Le besoin de mise à disposition rapide de l'information nécessite des méthodes automatiques et fiables pour le traitement de gros volumes de données issus de l'observation spatiale. Par exemple, les procédures actuelles de production de la base de données "Corine Land Cover", fondées sur la photo-interprétation, imposent des délais de mise à disposition importants, d'environ trois ans. Des techniques d'apprentissage automatique (Inglada et al., 2015) peuvent être utilisées aujourd'hui pour cartographier de façon détaillée et rapide l'occupation des sols à l'échelle nationale (figure 1), voire continentale.

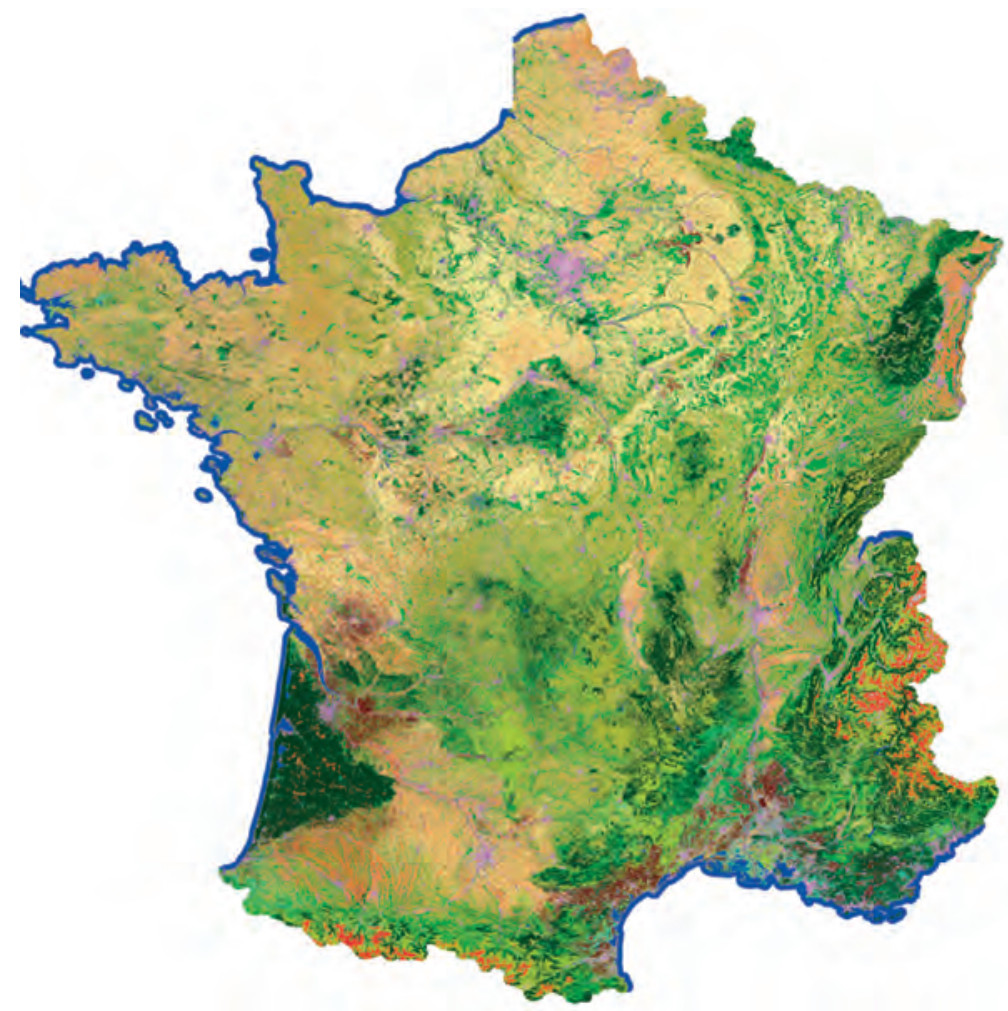

NoData

Culture d'été

Culture d'hiver

Forêt Feuilles Caduques

Forêt Feuilles Persistantes Pelouses

Estives-landes

Bâti dense

Bâti diffus

Autre bâti

Routes

Surfaces minérales

Plages et dunes

Eau

Glacier ou neiges éternelles

Prairie permanente

Verger

Vignes

Figure 1. Carte d'occupation des sols en France obtenue à partir d'images Landsat-8 acquises en 2014. 
Ainsi, dans le domaine optique, l'observation (après correction des effets atmosphériques) est une mesure de la réflectance de surface qui permet de suivre les variations saisonnières de la végétation et les propriétés des sols. Cette information de base est utilisée pour produire des variables biophysiques comme le LAI (figure 2) à même de caractériser l'état des couverts agricoles. Ces variables sont directement liées à la production primaire de la végétation et sont facilement calculables à partir de mesures de réflectances acquises dans le domaine optique. Un outil a été développé par le centre Inra d'Avignon (Baret et al., 2007), fondé sur la combinaison d'un modèle de transfert radiatif et d'un réseau de neurones pour calculer ces variables à partir de différents types de capteurs opérant dans l'optique. Cet outil, appelé Bvnet, est en accès libre sous la bibliothèque Orfeo toolbox (Inglada, 2015). Les observations dans le domaine de l'infrarouge thermique, via le suivi de la température de surface, permettent un suivi de l'état hydrique de la végétation. Les équipes de l'Inra et du Cesbio ont développé depuis plusieurs années des chaînes de traitement combinant des images opérant dans les domaines solaire et thermique pour cartographier l'évapotranspiration (en $\mathrm{mm}$ par jour) ou son équivalent énergétique (en $\mathrm{W} / \mathrm{m}^{2}$ ), le flux de chaleur latente. En particulier, GallegoElvira et al. (2013) ont développé le logiciel Evaspa qui fournit une estimation du flux de chaleur latente ainsi que l'incertitude associée à cette estimation. Ces cartographies de l'évapotranspiration contribuent à une meilleure quantification des besoins en eau des cultures, utiles par exemple pour mieux gérer l'irrigation. Elles constituent par ailleurs une information potentiellement utilisable pour la cartographie des propriétés hydrodynamiques des sols. Ces cartes sont validées à partir de mesures ponctuelles de LAI dérivées de photos hémisphériques traitées par le logiciel Can-eye (http://www6.paca.inra.fr/ can-eye) et de flux turbulents faites au sol avec des anémomètres soniques 3D.

\section{Suivi des sécheresses}

Les modèles numériques de l'atmosphère utilisés pour la prévision du temps, la prévision saisonnière ou la simulation de l'évolution du climat comprennent un sous-modèle capable
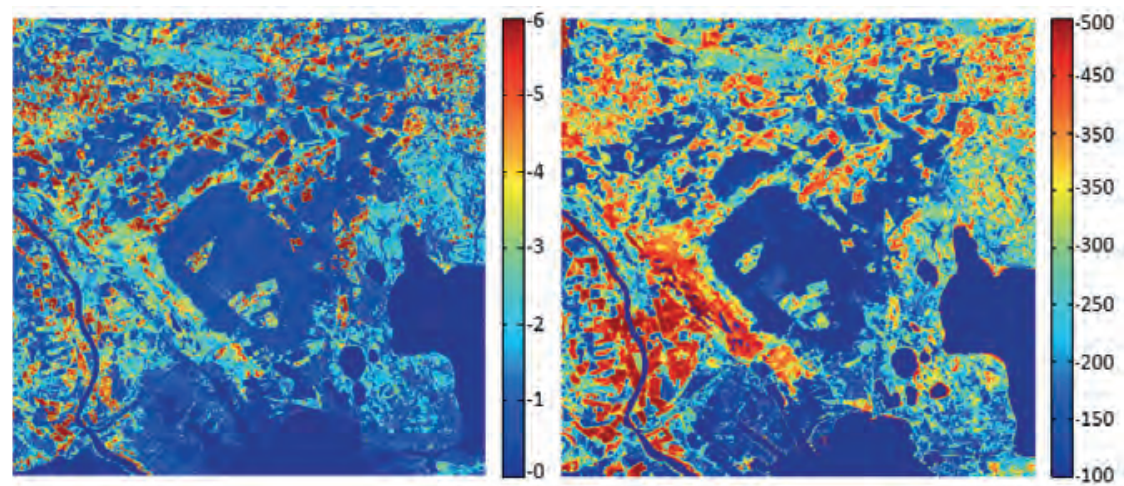

Figure 2. Cartographies obtenues à partir d'une image Landsat-7 acquise au-dessus de la région Crau-Camargue le 8 juillet 2008 de l'indice de surface foliaire à partir du logiciel Bvnet là gauche, en $\mathrm{m}^{2} \mathrm{~m}-2$ ), et du flux de chaleur latente à partir du logiciel Evaspa à $10 \mathrm{~h} 15$ en temps universel (à droite, en $\mathrm{W} \mathrm{m}^{-2}$, équivalent énergétique de l'évapotranspiration réelle). Dans les deux cas, les couleurs rouges correspondent aux couverts bien développés comme les zones de rizières ou les prairies irriguées qui présentent une végétation dense et évaporent beaucoup à cette période ; les zones en bleu correspondent aux parcelles récoltées en chaumes ou en sol nu et aux prairies sèches.

de décrire les échanges de matière et d'énergie entre les surfaces terrestres et l'atmosphère. En particulier, les processus physiques (par exemple l'évaporation, la diffusion de l'eau et de la chaleur dans le sol) et biologiques (par exemple la photosynthèse et la croissance des végétaux) du système sol-plante sont représentés dans ce modèle de surface, de façon explicite ou implicite selon la complexité du modèle. Les modèles des surfaces terrestres peuvent être utilisés en dehors des modèles atmosphériques dans diverses applications, en particulier le suivi de l'évapotranspiration, la simulation du débit des rivières et des ressources en eau, à plusieurs échelles spatiales. Dans ce contexte, ils sont alimentés par des variables atmosphériques de surface observées ou issues de modèles atmosphériques. Les simulations sont affectées par des erreurs liées aux incertitudes des forçages atmosphériques et aux incertitudes dans la représentation des processus.

Dans les applications climatologiques visant à reconstituer l'état du système sol-plante à un instant donné, il est intéressant de pouvoir agir sur le modèle de surface afin de corriger son écart aux observations satellitaires. Cela est indispensable afin de bien représenter l'impact des événements extrêmes comme les sécheresses agricoles. Aujourd'hui, les indicateurs de sécheresse agricole produits par Météo-France proviennent de simulations numériques de l'humidité du sol réalisées grâce à la plateforme de modélisation Surfex (surface externalisée) (Masson et al., 2013). Des recherches ont été menées afin d'évaluer l'impact de l'intégration de données satellitaires dans Surfex sur le suivi des sécheresses. Ainsi, un système d'assimilation de données a été mis en place (Barbu et al., 2014). Il permet de simuler la biomasse et le LAI des céréales à paille et des prairies naturelles sur la France. L'assimilation améliore la simulation de ces quantités par l'utilisation de produits satellitaires distribués gratuitement en temps quasi réel par le service Copernicus Global Land $^{1}$. La simulation est modifiée dès qu'une observation est disponible. Ces modifications sont appelées les incréments d'analyse. Dans le cas des céréales à paille, l'assimilation du LAI permet, par minimisation des incréments d'analyse, d'estimer la réserve utile des sols. Ce dernier paramètre correspond à la quantité maximale d'eau stockée dans le sol pouvant être utilisée par les plantes. La réserve utile est difficile à mesurer et le fait que l'on puisse l'estimer de façon indirecte à partir d'observations satellitaires de la végétation est un résultat remarquable.

Les observations du LAI sont donc particulièrement utiles dans ce contexte (voir encadré 2). L'utilisation de données de référence correspondant à des observations in situ des rendements agricoles montre que l'intégration de ces données satellitaires permet d'obtenir une corrélation significative entre la biomasse aérienne maximale annuelle simulée par Surfex et la production agricole à l'échelle d'un département.

1. http://land.copernicus.eu/global/ 


\section{Climat et rendement des cultures}

La production végétale est pénalisée par les déficits hydriques mais également par les excès d'eau. Il est souvent difficile pour les modèles du système sol-plante de représenter les effets des excès d'eau sur la production des céréales à paille car ils font intervenir des processus complexes (maladies, ravageurs, verse, etc.) dont le diagnostic est compliqué par les problèmes d'accessibilité des agriculteurs aux parcelles. Dans quelle mesure les produits satellitaires nous renseignent-ils sur la variabilité interannuelle des rendements ?

La comparaison entre les observations de LAI maximal annuel et les estimations in situ à l'échelle des départements français du rendement des céréales à paille montre une corrélation significative pour 30 départements, sur la période

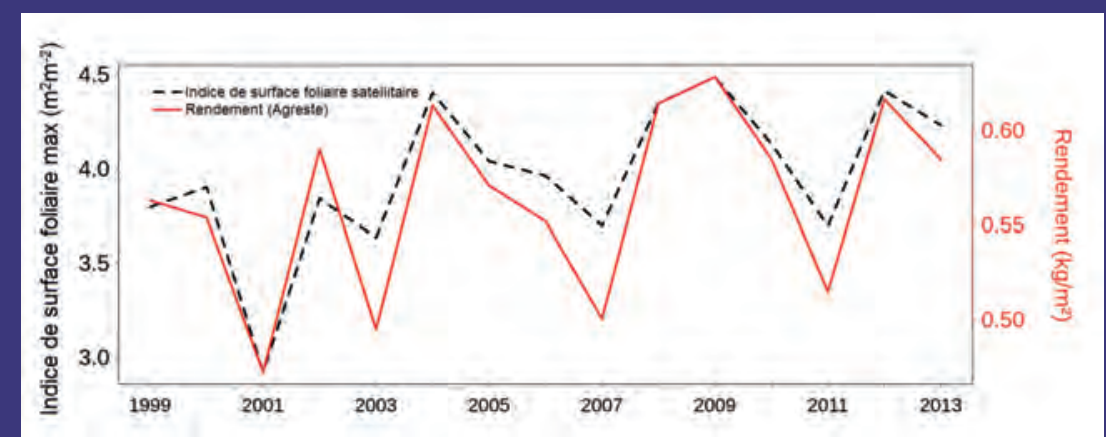

Figure 3. Moyennes pour 30 départements céréaliers français du maximum annuel d'indice de surface foliaire fourni par le service Copernicus Global Land (http://land.copernicus.eu/global/) à partir de données Végétation de Spot et des données de rendement des céréales à paille issues du serveur Agreste (http://agreste.agriculture.gouv.fr/).

Sans les données satellitaires, la corrélation est quasiment nulle. On montre également que de nouveaux indicateurs de sécheresse complémentaires de l'humidité du sol peuvent être construits en utilisant les simulations du LAI et de la biomasse aérienne, tous les dix jours. L'exemple du Puy-de-Dôme est présenté dans la
1999-2013. La figure 3 montre la moyenne annuelle de ces valeurs, dont la variabilité interannuelle est environ de $10 \%$. Une baisse des rendements agricoles et de LAI est observée en 2001, 2003, 2007 et 2011. Ces quatre années se caractérisent par des anomalies climatiques importantes. L'hiver 2000-2001 a été marqué par une pluviométrie excessive causant d'importantes inondations notamment dans le nord de la France.

L'ensoleillement a été déficitaire jusqu'au mois de mai 2001. Les années 2003 et 2011 ont été exceptionnellement chaudes, avec un printemps sec et des épisodes de canicules, particulièrement sévères en 2003. En 2007, après un printemps chaud et propice au développement de la végétation, l'été humide a eu un impact négatif sur les rendements. figure 4, car ce département comporte à la fois des céréales à paille et des prairies. Sur la période 2008-2013, les plus fortes anomalies négatives de LAI sont observées en 2011. Le printemps 2011 a été exceptionnellement sec et chaud et cet événement a eu un impact important sur le développement de la végétation. Dans un premier

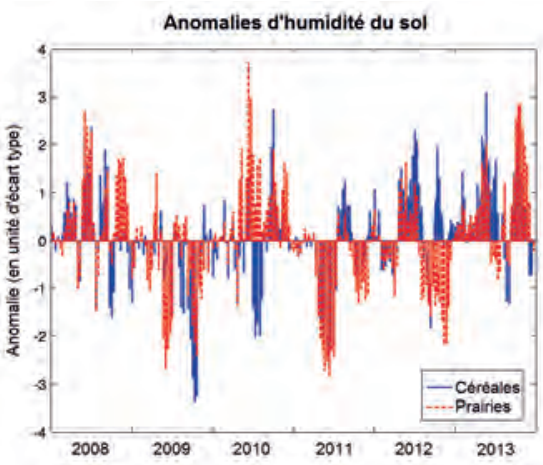

temps, les fortes températures et le fort éclairement ont favorisé la photosynthèse et conduit à des valeurs de LAI plus élevées que d'habitude, en particulier en avril. Dans un second temps, la sécheresse a provoqué un stress hydrique, ce qui a conduit à des valeurs anormalement basses de la photosynthèse et du LAI, de mai à juin. En revanche, juillet a été plus humide, ce qui a permis un redémarrage de la végétation. Au printemps, les indicateurs de sécheresse liés à la végétation ont un comportement différent de l'humidité du sol. En effet, les facteurs qui conduisent à un accroissement de la photosynthèse et à une croissance accrue de la végétation tendent à augmenter l'évaporation et donc à diminuer l'humidité du sol. L'impact négatif de la sécheresse sur la végétation apparaît plus tard que l'impact négatif sur l'humidité du sol, c'est-à-dire lorsque l'humidité du sol est trop faible pour permettre la croissance des plantes. Cette sécheresse intense, suivie de précipitations supérieures à la climatologie, montre que la variabilité de la croissance de la végétation d'une année sur l'autre a un impact sur les simulations de l'humidité du sol. La possibilité de produire conjointement des indicateurs de sécheresse liés à la végétation et à l'humidité du sol pourrait améliorer le suivi de l'impact des événements climatiques sur la production agricole.

\section{Flux et stocks de carbone}

\section{Agriculture et $\mathrm{CO}_{2}$}

La télédétection à haute résolution spatiale et temporelle, couplée à la modélisation, constitue un outil privilégié, car elle permet d'étudier de grands territoires tout en prenant

Figure 4. Anomalies décadaires en unités d'écart type de l'humidité du sol, de l'indice foliaire et de la biomasse aérienne, pour le Puy-de-Dôme dans une zone de céréales à paille (traits bleus) et dans une zone de prairies naturelles (traits rouges), de 2008 à 2013, après intégration de données satellitaires dans le modèle Surfex. 

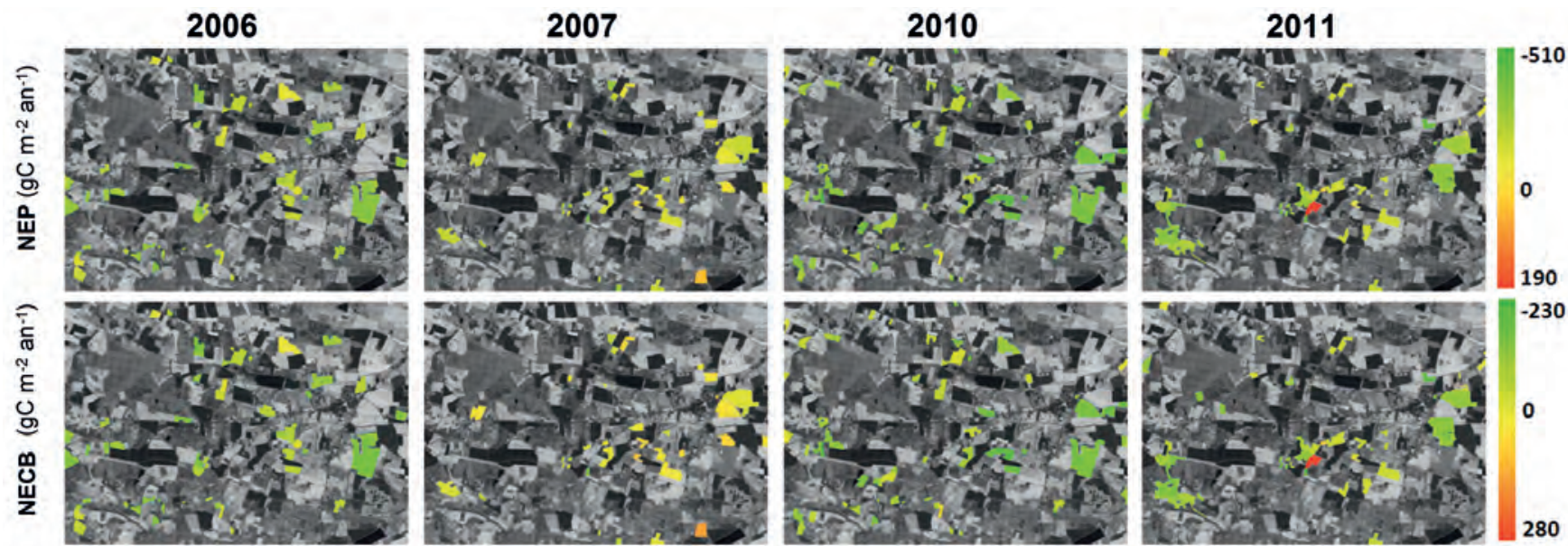

Figure 5. Flux nets annuels de $\mathrm{CO}_{2}$ (NEP) et bilans annuels de carbone (NECB, pour Net Ecosystem Carbon Budget) des cultures de blé d'hiver dans le sud-ouest de la France obtenus avec le modèle Safye-CO, (Veloso, 2014). Les données à hautes résolutions spatiale et temporelle des capteurs Formosat-2 et Spot ont été intégrées dans le modèle en produisant des cartes d'occupation du sol et de dynamique d'indice de surface foliaire.

en compte leur complexité et leur dynamique. Elle permet par exemple d'identifier les pratiques culturales (rotation des cultures, travail du sol, irrigation, ensilage...) à l'échelle de la parcelle agricole. Elle permet aussi d'assimiler dans les modèles de fonctionnement des cultures des paramètres biophysiques (LAI, biomasse, humidité du sol...) qui sont autant d'informations utiles voire indispensables en entrée de ces modèles.

Certains modèles de fonctionnement des cultures, comme Spa (Sus et al., 2010 ; Revill et al., 2013) ou Safye- $\mathrm{CO}_{2}$ (Veloso, 2014), ont été conçus pour intégrer des données satellitaires optiques ou radar à hautes résolutions spatiale et temporelle. Ils peuvent ainsi fournir une description spatialisée précise de la phénologie et du fonctionnement des cultures, et notamment des composantes du flux net de $\mathrm{CO}_{2}$ (photosynthèse, respiration du sol et de la plante) et d'une partie des composantes du bilan de carbone (BilanC). Le bilan de carbone annuel des cultures prend en compte les apports de carbone sous forme d'engrais organiques ( $\left.C_{\text {apports }}\right)$, les exports de carbone à la récolte ( $\left.C_{\text {exports }}\right)$ et le flux net annuel de $\mathrm{CO}_{2}$ entre l'agro-écosystème et l'atmosphère (Net Ecosystem Production, NEP) qui est la somme des flux nets de $\mathrm{CO}_{2}$ journaliers : BilanC = $\mathrm{NEP}+C_{\text {apports }}+C_{\text {exports. }}$ Le terme NEP est négatif si la parcelle est un puits annuel de $\mathrm{CO}_{2}$ (ce qui est souvent le cas pour les cultures d'hiver à long cycle), sinon il est positif (souvent le cas pour les cultures d'été), le terme $C_{\text {exports }}$ est de signe négatif et le terme $C_{\text {exports }}$ est positif. Le bilan de carbone sera donc négatif si l'écosystème se comporte comme un puits de carbone (stockage de carbone organique dans le sol) et il sera positif si c'est une source de carbone (déstockage de carbone organique dans le sol qui vient enrichir l'atmosphère en $\mathrm{CO}_{2}$ ). La figure 5 montre une série de bilans annuels de carbone dans une zone agricole du sud-ouest de la France, produite par Safye- $\mathrm{CO}_{2}$. Des séries temporelles de LAI sont utilisées pour l'étalonnage des paramètres phénologiques du modèle (jour de levée, début de sénescence, etc.). Le modèle est validé en termes d'estimations de biomasse, de rendement (exports de carbone à la récolte), de photosynthèse, de respiration de l'écosystème, de flux nets de $\mathrm{CO}_{2}$ et d'évapotranspiration, grâce à des observations in situ de biomasse et de flux acquises dans le cadre de l'Observatoire spatial régional ${ }^{2}$.

Cette approche nécessite d'avoir une estimation très précise de chacun des termes de BilanC pour obtenir des estimations correctes de ce dernier. En particulier, les termes NEP et $C_{\text {exports }}$ sont quantitativement les deux termes les plus importants, mais ils sont de signes opposés et ont tendance à se compenser. Pour obtenir des estimations précises de la NEP, il est essentiel de prendre en compte le développement des repousses et des plantes adventices, et la présence éventuelle de couverts intermédiaires. Le développement de ces végétaux en intercultures, observables par télédétection, permet de limiter les pertes de carbone des parcelles agricoles et donc d'augmenter le stockage de carbone (Ceschia et al., 2010). Cette approche de modélisation combinée aux données de télédétection pour l'estimation des bilans de carbone à grande échelle est soumise à deux limites méthodologiques. La première concerne la gestion des pailles après la récolte. À l'heure actuelle, il est très difficile, à partir des images de télédétection, de différencier les parcelles cultivées sur lesquelles la paille est exportée de celles sur lesquelles la paille est enfouie. En conséquence, les estimations de $C_{\text {exports }}$ présentent une large incertitude (qui se répercute sur BilanC). Des travaux s'appuyant sur une utilisation combinée de données multispectrales (optique, données hyperspectrales et radar) devront donc être menés pour tenter de fournir une information à la parcelle concernant la gestion des pailles. La deuxième concerne les apports d'engrais organiques. Puisqu'il n'est pas possible d'obtenir une estimation quantitative de cette variable à partir de données de télédétection, la solution actuelle pour estimer les apports de carbone organique à l'échelle des territoires réside dans l'utilisation de données d'inventaires régionaux ou nationaux.

\section{Fluorescence et photosynthèse}

La fluorescence chlorophyllienne est un rayonnement émis par la végétation dans le rouge et le proche infrarouge, susceptible de donner des informations sur son état physiologique, notamment son niveau de stress, et intrinsèquement lié à la photosynthèse. En effet, lorsque les photons sont absorbés par les chloroplastes des cellules végétales, leur énergie est soit utilisée pour la photosynthèse, soit dissipée sous forme de chaleur, notamment sous l'effet de mécanismes complexes de 
photoprotection, soit réémise sous forme de rayonnement à plus grande longueur d'onde : c'est la fluorescence. L'intérêt de la fluorescence pour le suivi de la végétation et de son état a été démontré depuis longtemps. Elle peut être mesurée au niveau de la feuille pour quantifier le rendement de conversion photochimique. Malgré le faible niveau de cette émission, sa détection au niveau de la canopée a été démontrée à l'aide de capteurs actifs (lidars) et passifs en exploitant les possibilités de discrimination offertes par les bandes d'absorption étroites du spectre solaire $\left(\mathrm{O}_{2}-\mathrm{A}, \mathrm{O}_{2}-\mathrm{B}, \mathrm{Ha}, \mathrm{K}\right.$, etc.) (Moya et al., 2004).

Si des produits satellitaires de fluorescence sont disponibles depuis longtemps pour les océans, ils n'existent que depuis 2011 sur les surfaces continentales. Ces produits sont dérivés des mesures des spectromètres à haute résolution Gosat/Tanso-FTS, Metop/ Gome 2 et plus récemment $O C O-2$, qui observent des concentrations de gaz atmosphériques. Ces produits de fluorescence sur les surfaces continentales ont montré une très forte corrélation avec la quantité de carbone captée par la photosynthèse continentale, appelée production primaire brute (Gross Primary Production, GPP) (Frankenberg et al., 2011). Cette information nouvelle a donc éveillé l'intérêt d'une communauté scientifique beaucoup plus large que les premiers utilisateurs de la fluorescence. Ces derniers ont beaucoup œuvré pour la préparation d'une mission centrée spécifiquement sur la fluorescence, la mission Flex (Fluorescence Explorer), qui a été sélectionnée fin 2015 par l'Agence spatiale européenne, avec un lancement prévu en $2022^{3}$. Le spectromètre imageur Floris permettra la mesure de la fluorescence dans le rouge et le proche infrarouge, en utilisant les bandes A et $\mathrm{B}$ de l'oxygène, ainsi que la mesure du PRI (Photochemical Reflectance Index), indicateur des processus de photoprotection. De plus, Flex volera en tandem avec Sentinel-3, qui fournira d'autres grandeurs comme le LAI ou le contenu en chlorophylle, formant une association unique à ce jour pour l'étude de la végétation continentale.

Le passage à une couverture globale des mesures de fluorescence a notamment attiré les modélisateurs des surfaces terrestres. En effet, la composante terrestre du cycle du carbone est la plus incertaine, et beaucoup de modèles numériques de la végétation reproduisent mal à la fois la répartition spatiale de la GPP et ses cycles saisonniers, ce qui est source d'erreur dans les projections climatiques. Étant donné le couplage entre les processus de fluorescence et de photosynthèse, les mesures de fluorescence peuvent donc être utilisées pour contraindre les modèles de surfaces continentales, via des techniques d'assimilation de données. Le préalable est d'introduire un modèle direct de la fluorescence au niveau de la feuille et au niveau de la canopée dans les modèles de surfaces continentales, incluant à la fois la modélisation du processus de couplage entre fluorescence et photosynthèse et le transfert radiatif. En effet, les mesures de fluorescence à l'échelle de la canopée intègrent des effets physiologiques et des effets structuraux liés à l'architecture de la canopée, avec notamment des effets directionnels et une forte réabsorption dans le rouge à la fois dans la feuille et dans le couvert. Les modélisateurs peuvent entre autres s'appuyer d'une part sur le modèle Scope (Van der Tol et al., 2009), qui possède ces fonctionnalités et a été développé dans le cadre de la préparation à la mission Flex, et d'autre part sur le développement de réseaux de mesures in situ de la fluorescence, du PRI et d'autres indices optiques de végétation couplés aux mesures d'échanges gazeux et d'énergie.

\section{Biomasse des forêts}

La biomasse des forêts est une information clé concernant la composante surface continentale du système Terre, agissant sur le climat à travers le cycle du carbone. Rappelons que la biomasse végétale est définie comme la masse de matière végétale sèche par unité de surface et qu'elle contient environ $50 \%$ de carbone. L'essentiel de la biomasse végétale se situe dans les forêts, et la majeure partie de ces dernières sont situées sous les tropiques. La forêt est à la fois un puits de carbone piégeant du $\mathrm{CO}_{2}$ atmosphérique pour le transformer en biomasse par photosynthèse, mais aussi une source de $\mathrm{CO}_{2}$, à cause en particulier de la déforestation causée par l'homme ou par les feux naturels. La distribution spatiale et l'ampleur des puits et des

\section{2. www.cesbio.ups-tlse.fr/fr/osr.html}

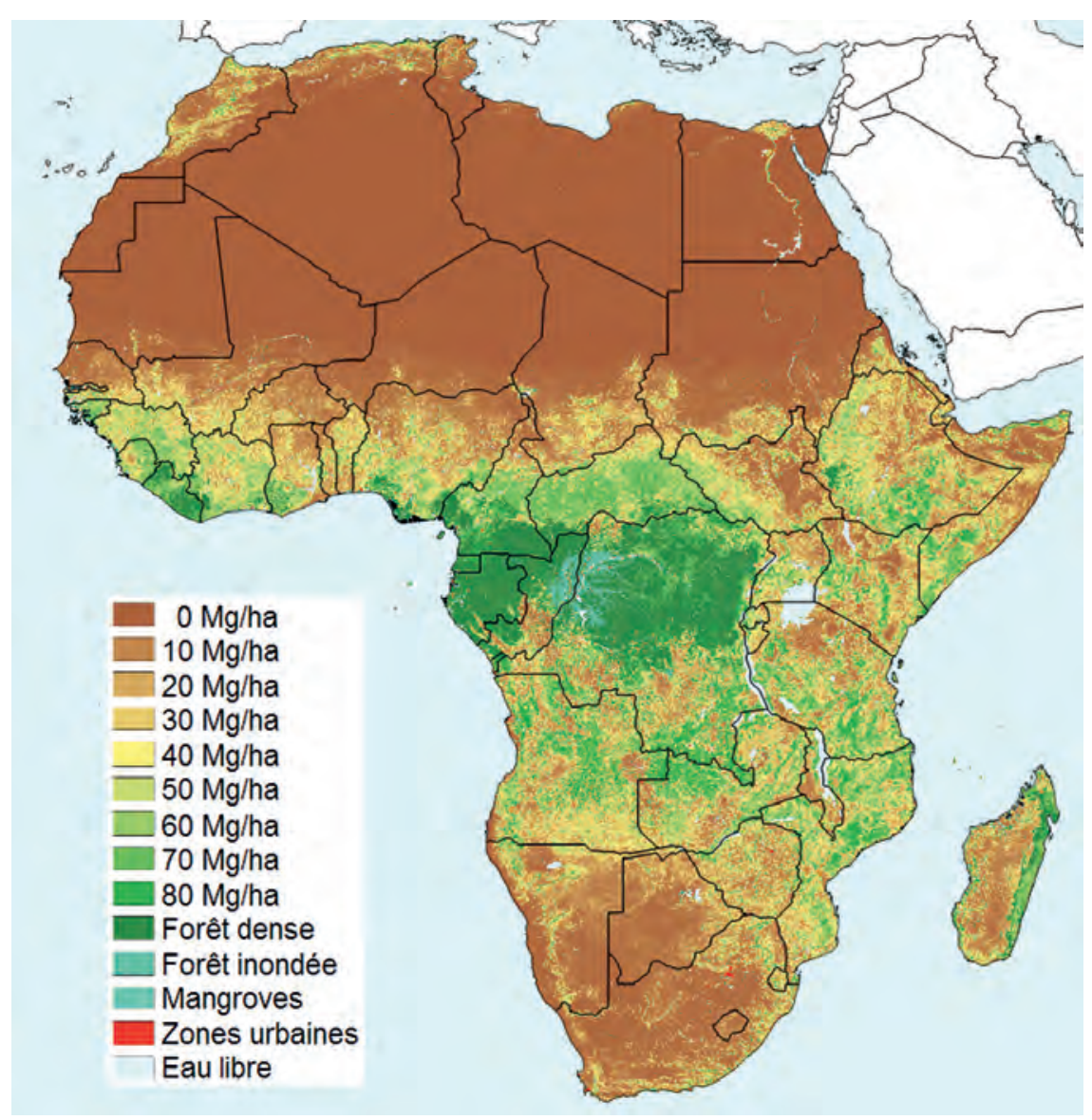

Figure 6. Biomasse aérienne, en $\mathrm{Mg} \mathrm{ha}^{-1}$ (ou $\mathrm{tha}^{-1}$ ), des forêts sèches et des savanes arborées en Afrique. La carte a une résolution spatiale de $25 \mathrm{~m}$ et est dérivée des données de l'instrument Palsar à bord d'Alos acquises en 2010. 
sources de $\mathrm{CO}_{2}$ sont toutefois très mal connues, en absence d'informations réactualisées sur les pertes et les gains de biomasse. Pour comprendre, atténuer et s'adapter au changement climatique, nous devons mesurer et suivre la biomasse forestière à l'échelle du globe. Cela permet de : i) réduire les incertitudes sur les estimations de stocks et des flux de carbone terrestre ; ii) d'avoir une référence objective dans l'application des traités internationaux, comme par exemple le programme Redd+ des Nations unies; iii) de contraindre les modèles de surface et du climat. Les mesures de biomasse sont donc essentielles pour le climat, elles sont aussi indispensables pour améliorer le suivi des ressources et de la biodiversité de la planète.

À l'échelle globale, seule l'observation par satellite pourrait assurer la cohérence et la fourniture régulière dans le temps d'estimations de biomasse. Les instruments optiques à bord de Landsat et Spot, ou Modis sur Aqua et Terra, sont pertinents pour la cartographie des écosystèmes forestiers, mais ont un intérêt limité pour les estimations de la biomasse. Opérant aussi dans le visible, mais à l'aide d'un faisceau de lumière émise par un laser, le lidar permet d'estimer la hauteur des couverts. L'instrument Glas sur le satellite Icesat a fourni dans le passé des données très intéressantes. Une suite est en préparation avec l'instrument Gedi sur Icesat-2. Les mesures lidar depuis l'espace produisent aux passages du satellite des transects discontinus. Estimer la biomasse à partir de ces données demande des informations supplémentaires pour transformer les hauteurs en valeurs de biomasse et pour l'extension spatiale des surfaces échantillonnées. Pour ces raisons, les cartes de biomasse obtenues aujourd'hui avec ces méthodes sont entachées d'importantes incertitudes.

La télédétection par radar à synthèse d'ouverture SAR (synthetic aperture radar) est fondée sur la rétrodiffusion des ondes radar qui pénètrent dans un volume de couvert forestier et qui interagissent avec les diffuseurs contenus dans ce volume que sont les feuilles, les tiges, les branches et les troncs. Le signal rétrodiffusé est indirectement lié à la biomasse de ces diffuseurs. La longueur d'onde du radar a un impact déterminant sur la gamme de biomasse qui peut être estimée. Parmi les systèmes actuels, seuls les SAR opérant en bande L (longueur d'onde d'environ $25 \mathrm{~cm}$ ), comme l'instrument Palsar ${ }^{4}$ à bord du satellite $A l o s^{5}$ et Palsar-2 à bord du satellite Alos-2, permettent d'estimer la biomasse jusqu'à une limite d'environ 100 tonnes par hectare (Mermoz et al., 2014). Cette limite correspond à des forêts peu denses et il est possible de cartographier la biomasse des forêts sèches, en régénération ou des savanes arborées. $\mathrm{La}$ figure 6 montre une carte de biomasse de telles formations végétales en Afrique, dérivée des données de Alos-Palsar.

Pour les forêts tropicales, qui ont un impact considérable sur le bilan de carbone terrestre, il n'existe pas aujourd'hui d'instrument en orbite permettant de sonder les couverts forestiers de 40-50 $\mathrm{m}$ de hauteur, dont la biomasse peut atteindre 500 tonnes par hectare. Les recherches entreprises dans ce domaine depuis une vingtaine d'années ont montré que seul un radar opérant à la plus basse fréquence possible depuis l'espace pourrait avoir de telles performances. C'est ainsi que la mission Biomass avec un SAR en bande $\mathrm{P}$ (longueur d'onde $68 \mathrm{~cm}$ ) a été proposée et sélectionnée par l'Agence spatiale européenne, pour un lancement prévu en 2021 (Le Toan et al., 2011). Les données seront exploitées par un ensemble de méthodes avancées (polarimétrie, interférométrie et tomographie) pour fournir des estimations de biomasse et de hauteur des couverts, et un suivi de leur changement tous les 6 mois. La résolution spatiale (de $200 \mathrm{~m}$ ) et les incertitudes des produits $( \pm 20 \%$ en biomasse) sont définies pour être compatibles avec les besoins des traités internationaux et avec les requis des modèles de carbone terrestre.

\section{Conclusion}

De plus en plus de produits satellitaires permettant de suivre le développement de la végétation à l'échelle mondiale sont disponibles librement, en temps quasi réel ou sous forme de longues séries temporelles couvrant plusieurs décennies. Cela permet de vérifier les modèles des surfaces terrestres, mais aussi de les améliorer par l'étalonnage de paramètres clés ou par l'intégration des observations par assimilation de données. L'arrivée en accès libre de données à haute répétitivité temporelle et à haute résolution spatiale (décamétriques) va permettre d'appliquer à des échelles régionales et locales des méthodes d'assimilation de données déjà mises en œuvre à l'échelle mondiale. Cela est particulièrement utile pour le suivi des zones agricoles : les récentes missions spatiales (comme Sentinel-1, Sentinel-2, et Landsat-8) offrent des perspectives intéressantes, grâce à leur fauchée élargie associée à une courte période de revisite et de nombreuses bandes spectrales, qui permettent le suivi des changements rapides de la végétation et de l'état du sol au sein même des parcelles agricoles. Il sera plus facile de représenter dans les modèles la diversité des cultures et les assolements. Une voie d'amélioration pour le suivi des zones agricoles réside dans l'exploitation de combinaisons entre instruments opérant dans des gammes de longueurs d'onde différentes. L'estimation du LAI à partir de données de télédétection radar (ou optique et radar), qui sont moins sujettes à saturation que les données optiques pour des fortes valeurs de LAI, permettrait de mieux étalonner les paramètres des modèles (Revill et al., 2013). La mission Flex permettra d'observer la fluorescence afin de caractériser la photosynthèse à des échelles hectométriques, alors que les produits actuels sont disponibles à très basse résolution spatiale (de 20 à $50 \mathrm{~km}$ ). Enfin, la biomasse des forêts, qui est une variable essentielle pour le climat, est l'objet de travaux visant à exploiter les données des instruments actuels (lidar, SAR) et aura dans quelques années une mission spatiale dédiée (Biomass).

\section{Remerciements}

Les travaux portant sur l'assimilation de données dans Surfex ont été cofinancés par la Commission européenne dans le cadre du projet Imagines (FP7 311766). Les travaux de thèse d'Hélène Dewaele sont cofinancés par le Cnes et MétéoFrance. Les travaux du Laboratoire des sciences du climat et de l'environnement et du Laboratoire de météorologie dynamique sur la fluorescence en lien avec la mission Flex sont cofinancés par le Cnes (projets Tosca Fluor et Actipass) et l'ESA.

\footnotetext{
3. http://earth.esa.int

4. Phased-Array L-band Synthetic Aperture Radar.

5. Advanced Land Observing Satellite, aussi appelé Daichi.
} 


\section{Bibliographie}

Barbu A.L., Calvet J.-C., Mahfouf J.-F., Lafont S., 2014. Integrating ASCAT surface soil moisture and GEOV1 leaf area index into the SURFEX modelling platform: a land data assimilation application over France. Hydrol. Earth Syst. Sci., 18, 173-192. doi: 10.5194/hess-18-173-2014

Baret F., Hagolle 0., Geiger B., Bicheron P., Miras B., Huc M., Berthelot B., Nino F., Weiss M., Samain O., Roujean J.-L., Leroy M., 2007. LAl, fAPAR and fCover CYCLOPES global products derived from VEGETATION — Part 1: Principles of the algorithm. Remote Sens. Environ., 110, 275-286. doi: 10.1016/j.rse.2007.02.018

Bojinski S., Verstraete M., Peterson T.C., Richter C., Simmons A., Zemp M., 2014. The concept of essential climate variables in support of climate research, applications, and policy. Bull. Am. Meteorol. Soc., 95, 1431-1443.

Ceschia E., Béziat P., Dejoux J.-F., Aubinet M., Bernhofer C., Bodson B., Buchmann N., Carrara A., Cellier P., Di Tommasi P., Elbers J.A., Eugster W., Grünvald T., Jacobs C.M.J., Jans W.W.P., Jones M., Kutsch W., Lanigan G., Magliulo E., Marloie O., Moors E.J., Moureaux C., Olioso A., Osborne B., Sanz M.J., Saunders M., Smith P., Soegaard H., Wattenbach M., 2010. Management effects on net ecosystem carbon and GHG budgets at European crop sites. Agr. Ecosyst. Environ., 139, 363-383. doi: 10.1016/j.agee.2010.09.020.

Frankenberg C., Fisher J.B., Worden J., Badgley G., Saatchi S.S., Lee J.E., Toon G.C., Butz A., Jung M., Kuze A., Yokota T., 2011. New global observations of the terrestrial carbon cycle from GOSAT: Patterns of plant fluorescence with gross primary productivity. Geophys. Res. Lett., 38, 1-6. doi: 10.1029/2011GL048738.

Gallego-Elvira B., Olioso A., Mira M., Reyes-Castillo S., Boulet G., Marloie O., Garrigues S., Courault D., Weiss M., Chauvelon P., Boutron 0., 2013. EVASPA (EVapotranspiration Assessment from SPAce) tool: An overview, four decades of progress in monitoring and modeling of processes in the soil-plant-atmosphere system: applications and ahallenges. Procedia Environ. Sci., 19, 303-310. doi: 10.1016/.jproenv.2013.06.035.

Inglada J., 2015. Biophysical vegetation variable estimation (LAI, fAPAR, fCover) from optical satellite imagery by machine learning inversion of a radiative transfer model, open-access software. doi:10.5281/zenodo.35506.

Inglada J., Arias M., Tardy B., Hagolle O., Valero S., Morin D., Dedieu G., Sepulcre G., Bontemps S., Defourny P., Koetz B., 2015. Assessment of an operational system for crop type map production using high temporal and spatial resolution satellite optical imagery. Remote Sens., 7, 12356-12379.

Le Toan T., Quegan S., Davidson M., Balzter H., Paillou P., Papathanassiou K., Plummer S., Saatchi S., Shugart H., Ulander L., 2011. The BIOMASS mission: Mapping global forest biomass to better understand the terrestrial carbon cycle. Remote Sens. Environ., 115, 2850-2860.

Masson V., Le Moigne P., Martin E., Faroux S., Alias A., Alkama R., Belamari S., Barbu A., Boone A., Bouyssel F., Brousseau P., Brun E., Calvet J.-C., Carrer D., Decharme B., Delire C., Donier S., Essaouini K., Gibelin A.-L., Giordani H., Habets F., Jidane M., Kerdraon G., Kourzeneva E., Lafaysse M., Lafont S., Lebeaupin-Brossier C., Lemonsu A., Mahfouf J.-F., Marguinaud P., Mokhtari M., Morin S., Pigeon G., Salgado R., Seity Y., Taillefer F., Tanguy G., Tulet P., Vincendon B., Vionnet V., Voldoire A., 2013. The SURFEXv7.2 land and ocean surface platform for coupled or offline simulation of earth surface variables and fluxes. Geosci. Model Dev., 6, 929-960. doi: 10.5194/gmd-6-929-2013.

Mermoz S., Le Toan T., Villard L., Réjou-Méchain M., Seifert-Granzin J., 2014. Biomass assessment in the Cameroon savanna using ALOS PALSAR data. Remote Sens. Environ., 155, 109-119.

Moya I., Camenen L., Evain S., Goulas Y., Cerovic Z.G., Latouche G., Flexas J., Ounis A., 2004. A new instrument for passive remote sensing 1. Measurements of sunlight-induced chlorophyll fluorescence. Remote Sens. Environ., 91, 186-197.

Revill A., Sus 0., Barrett B., Williams M., 2013. Carbon cycling of European croplands: A framework for the assimilation of optical and microwave Earth observation data. Remote Sens. Environ., 137, 84-93.

Sus 0., Williams M., Bernhofer C., Béziat P., Buchmann N., Ceschia E., Doherty R., Eugster W., Grünvald T., Kutsch W., Smith P., Wattenbach M., 2010. A linked carbon cycle and crop developmental model: Description and evaluation against measurements of carbon fluxes and carbon stocks at several European agricultural sites. Agr. Ecosyst. Environ., 139, 402-418.

Van der Tol C., Verhoef W., Timmermans J., Verhoef A., Su Z., 2009. An integrated model of soil-canopy spectral radiances, photosynthesis, fluorescence, temperature and energy balance. Biogeosciences, 6, 3109-3129.

Veloso A., 2014. Regional estimates of the production, fluxes and budgets of carbon and water for winter wheat by using high resolution remote sensing data combined with a crop model: Application to southwest France, thèse de doctorat, Université Paul-Sabatier, Toulouse. 